\title{
Article \\ Human Reaction Times: Linking Individual and Collective Behaviour Through Physics Modeling
}

\author{
Juan Carlos Castro-Palacio ${ }^{1,+}{ }^{\oplus}$, Pedro Fernández-de-Córdoba ${ }^{2}{ }^{(}$, J. M. Isidro ${ }^{2}\left(\mathbb{D}\right.$, Sarira Sahu ${ }^{3} \mathbb{C}$ and \\ Esperanza Navarro-Pardo ${ }^{4, *} *$
}

1 Department of Earth Science and Engineering, Imperial College London, London SW7 2AZ, UK; juancas@upvnet.upv.es

2 Instituto Universitario de Matemática Pura y Aplicada, Universitat Politècnica de València, 46022 Valencia, Spain; pfernandez@mat.upv.es (P.F.-d.-C); joissan@mat.upv.es (J.M.I.)

3 Instituto de Ciencias Nucleares, Universidad Nacional Autónoma de México, Circuito Exterior, C.U., A. Postal 70-543, Mexico DF 04510, Mexico; sarira@nucleares.unam.mx

4 Department of Developmental and Educational Psychology, Universitat de València, 46010 Valencia, Spain

* Correspondence: esperanza.navarro@uv.es

+ Current address: Department of Electrical Engineering, Electronics, Automation, and Applied Physics, Technical University of Madrid, 28012 Madrid, Spain.

Citation: Castro-Palacio, J.C.; Fernández de Córdoba, P.; Isidro, J.M.; Sahu, S.; Navarro-Pardo, E. Human Reaction Times: Linking Individual and Collective Behaviour Through Physics Modeling. Symmetry 2021, 13, 451. https://doi.org/10.3390/sym 13030451

Academic Editor: Vincenzo Vespri

Received: 20 February 2021

Accepted: 5 March 2021

Published: 10 March 2021

Publisher's Note: MDPI stays neutral with regard to jurisdictional claims in published maps and institutional affiliations.

Copyright: (c) 2021 by the authors. Licensee MDPI, Basel, Switzerland. This article is an open access article distributed under the terms and conditions of the Creative Commons Attribution (CC BY) license (https:/ / creativecommons.org/licenses/by/ $4.0 /)$.

\begin{abstract}
An individual's reaction time data to visual stimuli have usually been represented in Experimental Psychology by means of an ex-Gaussian function. In most previous works, researchers have mainly aimed at finding a meaning for the parameters of the ex-Gaussian function which are known to correlate with cognitive disorders. Based on the recent evidence of correlations between the reaction time series to visual stimuli produced by different individuals within a group, we go beyond and propose a Physics-inspired model to represent the reaction time data of a coetaneous group of individuals. In doing so, a Maxwell-Boltzmann-like distribution appeared, the same distribution as for the velocities of the molecules in an Ideal Gas model. We describe step by step the methodology we use to go from the individual reaction times to the distribution of the individuals response within the coetaneous group. In practical terms, by means of this model we also provide a simple entropy-based methodology for the classification of the individuals within the collective they belong to with no need for an external reference which can be applicable in diverse areas of social sciences.
\end{abstract}

Keywords: physical psychology; brain thermodynamics; reaction times; ideal gas theory; visual stimuli

\section{Introduction}

Understanding the processes of decision-making is a fundamental goal in the social, behavioral and cognitive sciences and reaction time (RT) to a specific stimulus is a measure of how quickly an organism can respond. In cognitive neuroscience research RT is widely used as a measure of information processing speed. RT is known to vary significantly across individuals, yet very little is known about the neural basis for this variability. The reaction times to visual stimuli by human play very important role in daily life and there exist a considerable amount of important work [1-6]. There is a wide variety of situations in which the collective behaviour of a group of individuals is relevant such as health [7], sports [8], industry [9], and behavioural economics and finances [10], to mention just a few examples. In Experimental Psychology, the RT data of an individual are usually represented in terms of an ex-Gaussian function with three parameters, denoted as $\mu, \sigma$, and $\tau$, coming from the convolution between an exponential and a Gaussian function. Reaction time distributions are positively skewed [11,12], hence, are not properly described by standard central tendency estimators, such as the mean and standard deviation [13]. In this respect, the ex-Gaussian function has been proven to optimally fit the probability distribution curves of the RT. 
The ex-Gaussian parameters derived from representing RT data are usually interpreted in connection with cognitive disorders as certain correlations are claimed to exist [14-20]. For instance, the most commonly diagnosed cognitive disorder in childhood affecting the RT is Attention Deficit and Hyperactivity Disorder (ADHD). In this respect, the exponential component $\tau$ is found to correlate with ADHD, specifically in male children [21]. The ADHD and autism spectrum disorder (ASD) among children aged 7-10 years is studied [22] to gain insights into the attentional fluctuations, related to increased reaction time variability. The use of the ex-Gaussian function to fit reaction time data has been used extensively in the literature [11-24]. On reviewing the previous works, we could not come across any clear and direct correlation between the ex-Gaussian parameters and cognitive disorders.

This work builds on the analysis and interpretations carried out to date although with a global and systemic view of the group of individuals. There is reported evidence of correlation between the reaction time series of different individuals within a coetaneous group, even when visual stimuli are free of cultural influences and responses to visual stimuli occur on a short time scale with no direct exchange of information between individuals [25]. Furthermore, relevant frequencies appear when the reaction times along the sequence of stimuli are analysed by Fast Fourier Transform [25,26]. These results suggest the existence of a system rather than single uncorrelated individual. Motivated by this experimental evidence, we construct a physics-inspired model for the group considering it as a system of individuals (a collective). By doing so, we believe to gain new insights into the reaction time data by analysing the reaction times of all individuals. We go from the ex-Gaussian function, commonly used to parameterize the response time data of an individual, to a more general representation using the moments of this distribution. Thereafter, based on the proven Gaussian distribution of the moments, the well known Maxwell-Boltzmann (MB) distribution can be constructed to represent the collective behaviour of a group of individuals, namely, the probability distribution of the individual responses within a group. We discuss the correspondence of our model with the Ideal Gas model [27] and introduce a one-to-one correspondence between a collective of individuals and a system of particles (molecules). Interestingly, our model can provide a simple classification methodology of the individuals within a collective they belong to, without a need for an external reference.

\section{Description of the Sample and the Experiments}

For this research, a sample of 168 children ( 84 males and 84 females) with ages between 8 and 10 years was taken. The children were chosen uniformly randomly and the mean age was 9.1 years, with a standard deviation of 0.9 years. The children who participated in the experiments attended a primary school in Valencia (Spain). All children were healthy, namely, presented no seizures, brain injury or any other neurological damage. All this relevant information was obtained from parents and school psychologists, who assessed all the children biannually.

For our study, we obtained all necessary consents and authorisations at all necessary levels, specifically, the school authorities, and the Regional Education Authority. This study was carried out in accordance with the recommendations of the Secretariat of Education of the Valencian Community. Also, the protocol was approved by the Government of Valencia (Generalitat Valenciana). The due written consents from the parents/legal guardians of the children were obtained in accordance with the Helsinki Declaration [28]. A priori, the participants were informed and properly explained about the procedures before the commencement of the experiment.

Computer-based experiments were carried out using the Window program DMDX [29] widely used in the community of experimental and cognitive psychologists [16,18,19,24,29-31]. Using this program, stimuli were presented to the participants and RTs recorded. The tasks applied in this work correspond to the child version of the Attentional Network Test (ANT Child) [32]. The ANT Child is a task designed to test three attentional networks (alerting, orienting, and executive control) [33] in children with a child-friendly version 
of the flanker task with alerting and orienting cues. The alerting network is assessed by changes in reaction time as a result of a warning signal. Orienting is related to changes in the RT indicating where the target will take place. Finally, the efficiency of the executive control is tested by asking the children to answer by pressing the keys in indicating left or right direction of an image placed at the centre in between neutral, congruous or incongruous flankers. These three networks are related among themselves [34,35]. During the experiment, each child was sitting in a separate and quiet place so that the independence among children while performing the experiment was guaranteed. Laptops bearing DMDX software were used. The stimuli were presented randomly to avoid order presentation effects. In this type of experiments, the individual reaction times take place in a very short period of time, typically within a few hundred milliseconds $(70-2500 \mathrm{~ms})$. The visual stimuli were very simple and uncorrelated to any cultural background or educational training. Specifically, each stimulus consisted of 5 fish aligned horizontally looking to the right or the left. The colour was black and the background was white. The objective of the task was to identify, in each trial, the direction of the central fish. There were three cases depending on the orientation of the fish around the central one. The neutral case was when there was only the central fish. The congruent case was where the surrounding fish were placed in the same direction as the central fish. The third case was where the surrounding fish were placed in the opposite direction with respect to the central one. If the central fish was facing right, the key labelled " $\mathrm{M}$ " should be pressed; if not, the key labelled "Z" should be pressed. A total of 144 stimuli were presented in a random way and for a maximum of $2500 \mathrm{~ms}$ (maximum duration of a trial) or until the child pressed a key. The maximum duration of an experiment can be calculated as $144 \times 2500 \mathrm{~ms}=6 \mathrm{~min}$. This situation is only given when the participant does not respond to any of the visual stimuli which is a very unlikely situation. Overall, our experiments involved $144 \times 168=24,192$ reaction times. Figure 1 shows the probability distribution curves of the reaction times for each individual along with the average over all participants and the most likely value of the mean curve peaks around $550 \mathrm{~ms}$.

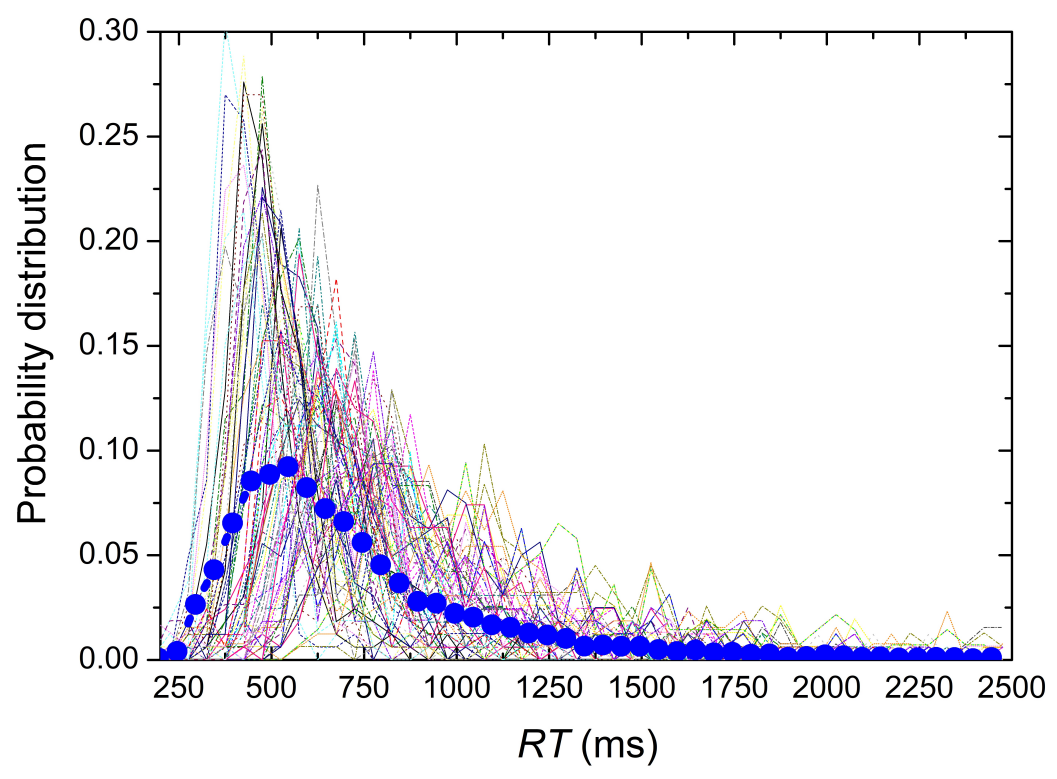

Figure 1. Probability distributions of the individuals response times and average curve (blue dotted line). 


\section{Results and Discussion}

Ex-Gaussian representation of reaction times. - The reaction time data derived from the computarized experiment is parametrized with the ex-Gaussian function. The first three moments of the ex-Gaussian distribution are calculated and their Gaussian behavior is established across the sample. The ex-Gaussian probability distribution results from the convolution of a Gaussian function and an exponential decay function. Let $f_{i}(t)$ be the ex-Gaussian probability distribution of the response times of the $i$-th individual [12] defined as $f_{i}(t)=f\left(\vec{a}_{i} ; t\right)$, and components of the vector are $\vec{a}_{i}=\left(\mu_{i}, \sigma_{i}, \tau_{i}\right)$. Then $f_{i}(t)$ is defined as

$$
f_{i}(t)=\frac{1}{2 \tau_{i}} \exp \left(\frac{1}{2 \tau_{i}}\left(2 \mu_{i}+\frac{\sigma_{i}^{2}}{\tau_{i}}-2 t\right)\right) \operatorname{erfc}\left(\frac{\mu_{i}+\frac{\sigma_{i}^{2}}{\tau_{i}}-t}{\sqrt{2} \sigma_{i}}\right),
$$

where $\operatorname{erf} c$ is the complementary error function [36] with $\mu_{i}, \sigma_{i}$, and $\tau_{i}$ are the parameters of the Gaussian $\left(\mu_{i}\right.$ and $\sigma_{i}$ ) and exponential decay $\left(\tau_{i}\right)$ functions which are convoluted to obtain the ex-Gaussian. The index $i$ runs from $i=1, \ldots, N=168$. Every individual is submitted to a test that yields a set of reaction times whose distribution can be represented by an ex-Gaussian function. We will characterize the distribution of RTs of the $i$-th individual in a more general way through its moments rather than using $\mu_{i}, \sigma_{i}$, and $\tau_{i}$. We can consider the moments centered either at the origin (raw moments) or at the corresponding average (central moments). Thus

$$
M_{i} \equiv \int_{-\infty}^{\infty} t f_{i}(t) d t
$$

is the raw moment of order one, while

$$
S_{i}^{2} \equiv \int_{-\infty}^{\infty}\left(t-M_{i}\right)^{2} f_{i}(t) \mathrm{d} t
$$

is the variance or second central moment of the random variable $t$ with probability distribution $f_{i}(t)$ and centered at $M_{i}$. The positive square root of $S_{i}$ is the standard deviation and the skewness $\lambda_{i}$ of the distribution is defined as

$$
\lambda_{i} \equiv \frac{1}{S_{i}^{2}} \int_{-\infty}^{\infty}\left(t-M_{i}\right)^{3} f_{i}(t) \mathrm{d} t,
$$

that is, the centered moment of order three, divided by the square of the standard deviation. We have divided by $S_{i}^{2}$ instead of $S_{i}^{3}$ to keep the same time dimension (milliseconds) for all three moments.

The moments of the ex-Gaussian function have the following forms: $M=\mu+\tau$, $S^{2}=\sigma^{2}+\tau^{2}$ and $\lambda=2 \tau^{3} /\left(\sigma^{2}+\tau^{2}\right)$ which clearly demonstrate that $\mu, \sigma$, and $\tau$ are not the moments of the distribution rather parameters of the Gaussian and exponential functions that convolute to give the ex-Gaussian distribution [12]. Thus an ex-Gaussian distribution can be characterised through its three moments as a new vector defined as $\vec{b}_{i}=\left(M_{i}, S_{i}, \lambda_{i}\right)$. To keep the dimensionality of the vector $\vec{b}_{i}$ equals to three, we considered the first three moments only. Here, the experimental input is the set of all $N$ vectors $\vec{b}_{i}$ representing the $N$ individuals analysed. By centering the components of $\vec{b}_{i}$ about their mean we express them in dimensionless form as $\vec{v}_{i}=\left(\left(M_{i}-\bar{M}\right) / \bar{M},\left(S_{i}-\bar{S}\right) / \bar{S},\left(\lambda_{i}-\bar{\lambda}\right) / \bar{\lambda}\right)$, where the mean value is given as

$$
\bar{X}=\frac{1}{N} \sum_{i=1}^{N} X_{i}, X=M, S, \lambda .
$$

The components of $\vec{v}_{i}$ are to be distributed normally, that is, they obey a Gaussian distribution over the ensemble of $N$ individuals which are shown in Figure 2 where the panels $(\mathrm{a}-\mathrm{c})$ exhibit this property explicitly. At the 0.05 confidence level, the data of the three moments were found to be significantly drawn from a normally distributed data 
according to the Kolmogorov-Smirnov normality test [37]. Therefore, the sample size used in this work $(N=168)$ is enough to show the Gaussian behaviour of the distributions of $v_{i}^{(1)}, v_{i}^{(2)}$, and $v_{i}^{(3)}$. In fact, when we randomly choose a sample of 50 individuals out of 168 , the percentage of relative errors among the respective variances of $v_{i}^{(1)}, v_{i}^{(2)}$, and $v_{i}^{(3)}$ are within $3.6 \%$. The fittings in Figure 2 were carried out by using the non-linear fitting algorithm of Levenberg-Marquardt [38,39].
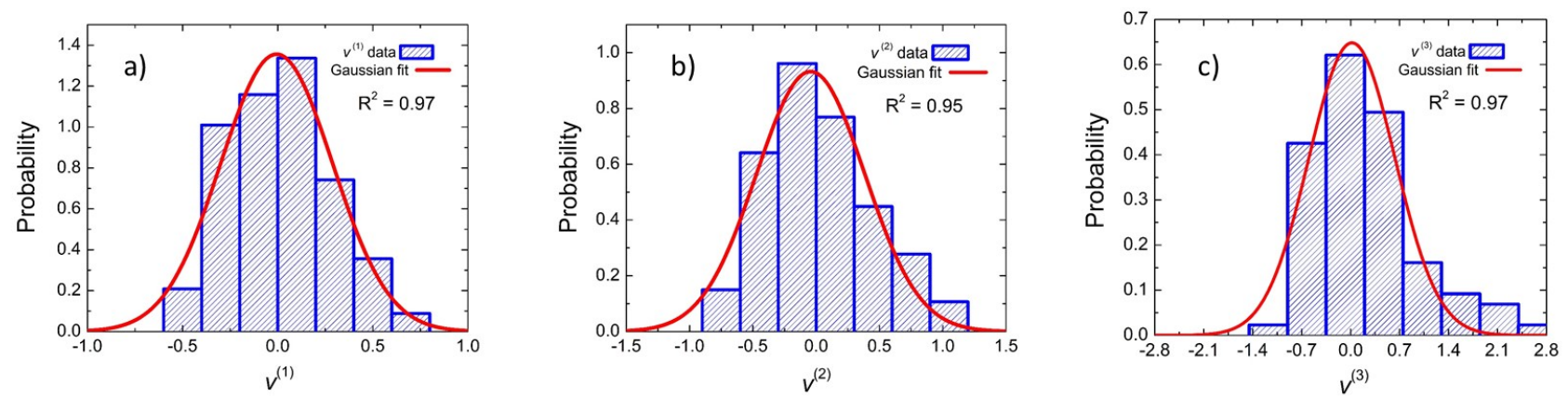

Figure 2. Probability distributions of the dimensionless moments calculated from 24,192 experimental reaction times over a sample of 168 children are shown in columns. The mean is shown in panel (a), the standard deviation is in panel (b) and the skewness is in panel (c). The $R^{2}$ coefficients of the fittings (red solid lines) are also shown.

The Ideal Gas model and reaction times. - In statistical physics, an Ideal Gas is an idealised gas made up of independent molecules/particles which move randomly and undergo elastic collisions. The velocities of the particles in the gas obey the Maxwell-Boltzmann (MB) probability density distribution [27],

$$
f(v, T)=4 \pi\left(\frac{m}{2 \pi k_{B} T}\right)^{3 / 2} v^{2} \exp \left(-\frac{m v^{2}}{2 k_{B} T}\right),
$$

where $v$ is the modulus of the particle velocity whose components are Gaussian-distributed with equal variance and centered about zero. The parameters $k_{B}, m$ and $T$ are respectively the Boltzmann constant, particle mass and temperature of the system in thermodynamic equilibrium. The MB distribution is a Chi distribution with three degrees of freedom and equal variances in general [40].

The entropy of a thermodynamic system is maximal in equilibrium. By imposing the constraint that $f(v, T)$ has a given finite variance, the MB probability density is the one that maximises the Boltzmann entropy denoted by $S$ and is defined as $S=k_{B} \ln W$, where $W$ is the number of microstates compatible with a given macrostate of the Ideal Gas. The ideal gas approximation can be used to study the present problem, where, we have $\left|\vec{v}_{i}\right|=\sqrt{\sum_{j=1}^{3}\left(v_{i}^{(j)}\right)^{2}}$. For our convenience, the MB probability density of Equation (6) is written as

$$
f(v, B)=\sqrt{\frac{2}{\pi}} B^{-3 / 2} v^{2} \exp \left(-\frac{v^{2}}{2 B}\right),
$$

where $B$ is the only free parameter. Using this, the Boltzmann entropy $S$ can be written as [27]

$$
\mathcal{S}=-\sum_{i=1}^{\mathrm{N}} p_{i} \ln p_{i}
$$

Here, $\mathrm{N}$ is the number of individuals in the sample, and $p_{i}$ is the probability associated to the $\mathrm{i}$-th individual given by $p_{i}=f\left(\left|\vec{v}_{i}\right| ; B\right) / \sum_{\mathrm{j}=1}^{\mathrm{N}} f\left(\left|\vec{v}_{j}\right| ; B\right)$. Apart from physics, entropy is also an analysis tool in a variety of fields [41-44], for example, in psychology it is widely used to analyse cognition experiments [41,42]. The panel a) of Figure 3 shows the fit of the MB distribution Equation (7) to all the RT experimental data for which $B=0.159 \pm 0.010$ $\left(R^{2}=0.88\right)$ and in panel $\left.\mathrm{b}\right)$ we show the entropy density curve. The Gaussian behaviour of 
the distributions of $v_{i}^{(1)}, v_{i}^{(2)}$, and $v_{i}^{(3)}$ leads to a Maxwell-Boltzmann distribution of $\left|\vec{v}_{i}\right|$, even if in our case, the variances of the three components are not all equal [40]. As already mentioned, we have obtained a coefficient of determination of $R^{2}=0.88$ using 24,192 experimental reaction times. Figure 3, panel (b) shows the entropy density curve for the experimental data of this work.
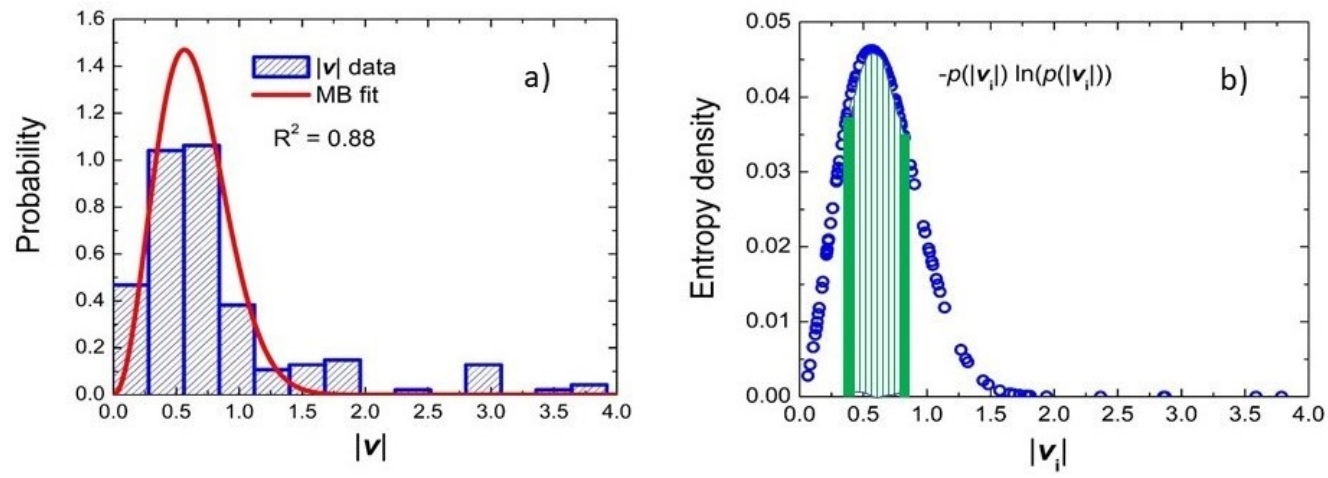

Figure 3. Maxwell-Boltzmann-like distribution of the reaction times of a system of individuals in panel (a) and the corresponding entropy density in panel (b). Each participant is represented with a blue open circle. The data shown in this figure involved 24,192 experimental reaction times over a sample of 168 children. The coefficient of determination of the fit, $R^{2}$, is also shown. As an example, $50 \%$ of the children have been represented by a patterned region in panel (b).

In order to gain access to the collective RT behaviour of a group of individuals it is necessary to take into account the whole set of experimental reaction times. In this respect, we have found that the $\mathrm{MB}$ distribution can provide a good characterisation. Around the maxima of the MB distribution and of the entropy density in Figure 3 panels $(\mathrm{a}, \mathrm{b})$, respectively, the individuals with most frequent values of $v$ are located. This is very useful and relevant information for the analysis in many contexts such as behavioural economics or normative development in psychology, where a central aim is to get to know the behaviour of the group. At this point we have made the transition from the description of the RTs of an individual, expressed in terms of the $N$ vectors $\vec{v}_{i}$, to collective description in terms of the parameter $B$. Each individual is represented now by a single scalar value $\left(\left|\vec{v}_{i}\right|\right)$.

Even when the entropy is a property of the full distribution, single individuals can be traced through, to determine their location at the entropy density curve, Figure 3 panel (b) and determine their contribution to the collective. In this fashion, we can know about their behaviour in relation to the group they belong to. No external reference is needed but the distribution of the collective as shown in panel (b) of Figure 3. Based on the contribution of each individual to the entropy density, a new classification methodology can in principle be established and from it the less and the more entropic individuals inside a group can be distinguished. When we say, "more or less entropic individuals", we refer to individuals contributing in a less or greater extent to the entropy density depending on the resulting value for each individual's term in Equation (8). We would like to emphasise that this entropy-based classification methodology is not an arbitrarily-defined criterion but a manifestation of a natural behaviour, which is the most important finding of this work.

The system of $N$ individuals is basically characterised by the parameter $B$ in Equation (7). As we have remarked previously, our sample consists of individuals who perform the experiments independently and respond to the visual stimuli in a very short time. This suggests to model the group of individuals as the particles in an Ideal Gas colliding elastically among themselves. So, there is a one-to-one correspondence between the time response of a group of children and a system of particles forming an ideal gas. The individual can be viewed as a particle while the three moments of the ex-Gaussian distribution for each child are analogous to the three components of the velocity vector of a 
particle. The distribution of the modulus of a vector defined over the moments leads to a Chi distribution with three degrees of freedom which is the Maxwell-Boltzmann distribution for an ideal gas. The particles form a thermodynamical system with an equilibrium temperature $T$. On the other hand, a collective is characterised by a parameter $B$, yet to come up with a physical interpretation of it in the present context. With these results we are moving to another level of generality beyond the ex-Gaussians which characterise the RT of an individual, in order to capture a regularity in the collective time response of a group of children. Our results open paths in two directions: we can now know about the collective time response of a group of individuals and also about the behaviour of a single individual in relation to the group they belong to.

\section{Conclusions}

In this letter, we have discussed the appearance of the Ideal Gas theory when interpreting the RT data of a group of individuals. The ex-Gaussian function represents well the RT data of an individual, but it fails to provide a complete and comprehensive characterisation of the reaction times of a group of individuals. We have built a physics-inspired model to represent the RTs of a coetaneous group of individuals and considering that they form a system, that is, a collective [25]. In doing so, we found that the Maxwell-Bolzmann distribution is a very good candidate. To validate our model, we used 24,192 experimental reaction times, obtaining a coefficient of determination of $R^{2}=0.88$. Our results suggest that the same principle of maximum entropy which is valid for the Ideal Gas is also applicable for the response times of a group of individuals. We can predict the behaviour of an individual in relation to the group he/she belongs to. In practical terms, our proposal of an entropy-based classification of individuals is not an ad hoc criterion but a methodology that may comply with a general principle. The emergence of a MB distribution relates directly to the fact that the first three moments of the RT distributions follow Gaussian distributions among the group of children. It should be emphasized that the Gaussian distribution of the moments among the individuals is not part of a mathematical formalism but something driven by nature. We could also see that a formal correspondence at the level of main components can be established between the response times of a coetaneous group of individuals and the system of particles of an ideal gas. This result may be applicable in different walks of life, such mental disease diagnosis, decision making in behavioural economics, and finances, where defining a reference or control group is important. However, much more work is needed to establish such connections.

Author Contributions: Conceptualization and methodology design: J.C.C.-P., P.F.-d.-C., J.M.I., S.S., E.N.-P.; experiment development and data collection: P.F.-d.-C., E.N.-P.; data analysis and simulations: J.C.C.-P., P.F.-d.-C., J.M.I., E.N.-P., S.S. All authors have read and agreed to the published version of the manuscript.

Funding: This research was partially funded by grant number RTI2018-102256-B-100 (Spain). S. S. is partially supported by DGAPA-UNAM (Mexico) Project No. IN103019.

Institutional Review Board Statement: The study was conducted according to the guidelines of the Declaration of Helsinki, and in accordance with the recommendations of the Secretariat of Education of the Valencian Community. Also, the protocol was approved by the Government of Valencia (Generalitat Valenciana).

Informed Consent Statement: We obtained all necessary consents and authorisations at all necessary levels, which includes the school authorities and the Regional Education Authority. A priori, the participants were informed and properly explained about the procedures before the commencement of the experiment. Written informed consent was obtained from all subjects involved in the study and their parents/legal guardians, in accordance with the Helsinki Declaration.

Data Availability Statement: We will chose to exclude this statement as the study did not report any data. 
Acknowledgments: The authors thank Miguel Ángel García March, Daniele Tommasini, Alberto Conejero, Luisberis Velázquez, María F. López-Ramón, and Ismael Orquín for many fruitful discussions and to Jeff Tupholme for language editing. We sincerely acknowledge the psychologists from the Interdisciplinary Modelling Group InterTech (www.intertech.upv.es, accessed on 25 October 2020) who participated in the collection of experimental data. J. C. C. P acknowledges the hospitality of Imperial College London where a major part of his work was carried out.

Conflicts of Interest: The authors declare no conflict of interest.

\section{References}

1. Thorpe, S.; Fize, D.; Marlot, C. Speed of processing in the human visual system. Nature 1996, 381, 520. [CrossRef] [PubMed]

2. Krajbich, I.; Bartling, B.; Hare, T.; Fehr, E. Rethinking fast and slow based on a critique of reaction-time reverse inference. Nat. Commun. 2015, 6, 7455. [CrossRef]

3. Barinaga, M. Neurons put the uncertainty into reaction times. Science 1996, 274, 344 . [CrossRef]

4. Tuch, D.S.; Salat, D.H.; Wisco, J.J.; Zaleta, A.K.; Hevelone, N.D.; Rosas, H.D. Choice reaction time performance correlates with diffusion anisotropy in white matter pathways supporting visuospatial attention. Proc. Natl. Acad. Sci. USA 2005, $102,12212$. [CrossRef]

5. Colonius, H.; Diederich, A. Measuring multisensory integration: From reaction times to spike counts. Sci. Rep. 2017, 7, 3023. [CrossRef]

6. Ritchie, J.B.; Beeck, H.D. Using neural distance to predict reaction time for categorizing the animacy, shape, and abstract properties of objects. Sci. Rep. 2019, 9, 13201. [CrossRef]

7. Abbasi-Kesbi, R.; Memarzadeh-Tehran, H.; Deenm, H.J. Technique to estimate human reaction time based on visual perception. Healthc. Technol. Lett. 2017, 4, 73. [CrossRef] [PubMed]

8. Badau, D.; Baydil, B.; Badau, A. Differences among three measures of reaction time based on hand laterality in individual. Sports 2018, 6, 45. [CrossRef] [PubMed]

9. Ruhai, G.; Weiwei, Z.; Zhong, W. Research on the driver reaction time of safety distance model on highway based on fuzzy mathematics. In Proceedings of the IEEE International Conference on Optoelectronics and Image Processing (ICOIP), Haikou, China, 10-11 November 2010; Volume 2, p. 293.

10. Yamagishi, T.; Matsumoto, Y.; Kiyonari, T.; Takagishi, H.; Li, Y.; Sakagami, R.K.M. Response time in economic games reflects different types of decision conflict for prosocial and proself individuals. Proc. Natl. Acad. Sci. USA 2017, 114, 6394. [CrossRef] [PubMed]

11. Luce, R.D. Response Times: Their Role in Inferring Elementary Mental Organization; Oxford University Press: New York, NY, USA, 1986.

12. Moret-Tatay, C.; Gamermann, D.; Navarro-Pardo, E.; de Córdoba, P.F. ExGUtils: A python package for statistical analysis with the ex-Gaussian probability density. Front. Psychol. 2018, 9, 1.

13. Ratcliff, R.; Murdock, B.B. Retrieval processes in recognition memory. Psychol. Rev. 1976, 83, 190. [CrossRef]

14. Gmehlin, D.; Fuermaier, A.B.M.; Walther, S.; Debelak, R.; Rentrop, M.; Westermann, C.; Sharma, A.; Tucha, L.; Koerts, J.; Tucha, O.; et al. Intraindividual variability in inhibitory function in adults with ADHD. An ex-Gaussian approach. PLoS ONE 2014, 9, e112298. [CrossRef] [PubMed]

15. Adamo, N.; Hodsoll, J.; Asherson, P.; Buitelaar, J.K.; Kuntsi, J. Ex-Gaussian, frequency and reward analyses reveal specificity of reaction time fluctuations to ADHD and not autism traits. J. Abnorm. Child Psychol. 2019, 47, 557. [CrossRef]

16. Moret-Tatay, C.; Leth-Steensen, C.; Irigaray, T.Q.; Argimon, I.I.L.; Gamermann, D.; Abad-Tortosa, D.; Oliveira, C.; de Córdoba, P.F. The effect of corrective feedback on performance in basic cognitive tasks: An analysis of RT components. Psychol. Belg. 2016, 56, 370. [CrossRef]

17. Mira-Iglesias, A.; Navarro-Pardo, E.; Conejero, J.A. Power-law distribution of natural visibility graphs from reaction times series. Symmetry 2019, 11, 563. [CrossRef]

18. Moreno-Cid, A.; Moret-Tatay, C.; Irigaray, T.Q.; Argimon, I.I.L.; Murphy, M.; Szczerbinski, M.; Martínez-Rubio, D.; Beneyto-Arrojo, M.J.; Navarro-Pardo, E.; de Córdoba, P.F. The role of age and emotional valence in word recognition: An ex-Gaussian analysis. Stud. Psychol. 2015, 57, 83. [CrossRef]

19. Moret-Tatay, C.; Moreno-Cid, A.; Argimon, I.I.L.; Quarti-Irigaray, T.; Szczerbinski, M.; Murphy, M.; Vázquez-Martínez, A.; Vázquez-Molina, J.; Sáiz-Mauleón, B.; Navarro-Pardo, E.; et al. The effects of age and emotional valence on recognition memory: An ex-Gaussian components analysis. Scand. J. Psychol. 2014, 55, 420. [CrossRef]

20. Navarro-Pardo, E.; Navarro-Prados, A.B.; Moret-Tatay, D.G. Differences between young and old university students on a lexical decision task: Evidence through an ex- gaussian approach. J. General Psychol. 2013, 140, 251. [CrossRef]

21. Leth-Steensen, C.; Elbaz, Z.K.; Douglas, V.I. Douglas Mean response times, variability, and skew in the responding of ADHD children: A response time distributional approach. Acta Psychol. 2000, 104, 167. [CrossRef]

22. Shahar, N.; Teodorescu, A.R.; Karmon-Presser, A.; Anholt, G.E.; Meiran, N. Memory for action rules and reaction time variability in attention-deficit/hyperactivity disorder. Biol. Psychiatry Cogn. Neurosci. Neuroimag. 2016, 1, 132. [CrossRef]

23. Matzke, D.; Wagenmakers, E.-J. Psychological interpretation of the ex-Gaussian and shifted Wald parameters: A diffusion model analysis. Psychon. Bull. Rev. 2009, 16, 798. [CrossRef] [PubMed] 
24. Hernaiz-Guijarro, M.; Castro-Palacio, J.C.; Navarro-Pardo, E.; Isidro, J.M.; de Córdoba, P.F. A probabilistic classification procedure based on response time analysis towards a quick pre-diagnosis of student's attention deficit. Mathematics 2019, 7, 473. [CrossRef]

25. Iglesias-Martínez, M.E.; Hernaiz-Guijarro, M.; Castro-Palacio, J.C.; Fernández-de-Córdoba, P.; Isidro, J.M.; Navarro-Pardo, E. Machinery Failure Approach and Spectral Analysis to Study the Reaction Time Dynamics over Consecutive Visual Stimuli: An Entropy-Based Model. Mathematics 2020, 8, 1979. [CrossRef]

26. Castellanos, F.X.; Sonuga-Barke, E.J.S.; Scheres, A.; Martino, A.D.; Hyde, C.; Walters, J.R. Varieties of Attention-Deficit/Hyperactivity Disorder-Related Intra-Individual Variability. Biol. Psychiatry 2005, 57, 1416. [CrossRef]

27. Tolman, R. The Principles of Statistical Mechanics; Dover Publications Inc.: New York, NY, USA, 2003.

28. World Medical Association. Declaration of Helsinki: Ethical Principles for Medical Research Involving Human Subjects. J. Am. Med. Assoc. 2013, 310, 2191. [CrossRef] [PubMed]

29. Forster, K.I.; Forster, J.C. DMDX: A windows display program with millisecond accuracy. Behav. Res. Method. Instrum. Comput. 2003, 35, 116. [CrossRef]

30. Garaizar, P.; Vadillo, M.A.; López-de-Ipiña, D.; Matute, H. Measuring software timing errors in the presentation of visual stimuli in cognitive neuroscience experiments. PLoS ONE 2014, 9, e85108. [CrossRef]

31. Rastle, K.; Davis, M.H. On the complexities of measuring naming. J. Experiment. Psychol. Hum. Percept. Perform. 2002, $28,307$. [CrossRef]

32. Rueda, M.R.; Fan, J.; McCandliss, B.D.; Halparin, J.D.; Gruber, D.B.; Lercari, L.P.; Posner, M.I. Development of attentional networks in childhood. Neuropsychologia 2004, 42, 1029. [CrossRef] [PubMed]

33. Fan, J.; McCandliss, B.D.; Sommer, T.; Raz, A.; Posner, M.I. Testing the efficiency and independence of attentional networks. J. Cog. Neurosci. 2002, 14, 340. [CrossRef] [PubMed]

34. Posner, M.I.; Dehaene, S. Attentional networks. Trends Neurosci. 1994, 17, 75. [CrossRef]

35. Posner, M.I.; Raichle, M.E. Images of Mind; Scientific American Library: New York, NY, USA, 1994.

36. Abramowitz, M.; Stegun, I.A. (Eds.) Handbook of Mathematical Functions: With Formulas, Graphs, and Mathematical Tables; Dover Publications Inc.: New York, NY, USA, 1965.

37. Doob, J.L. Stochastic Processes; John Wiley \& Sons Inc.: Hoboken, NJ, USA, 1966.

38. Levenberg, K. A method for the solution of certain non-linear problems in least squares. Q. Appl. Math. 1944, 2, 164. [CrossRef]

39. Marquardt, D. An algorithm for least-squares estimation of nonlinear parameters. J. Soc. Ind. Appl. Math. 1963, 11, 431. [CrossRef]

40. Castro-Palacio, J.C.; Isidro, J.M.; Navarro-Pardo, E.; Velazquez, L.; Fernández-de-Córdoba, P. Monte Carlo Simulation of a Modified Chi Distribution with Unequal Variances in the Generating Gaussians. A Discrete Methodology to Study Collective Response Times. Mathematics 2021, 9, 77. [CrossRef]

41. Del-Prado-Martín, F. Macroscopic thermodynamics of reaction times. J. Math. Psychol. 2011, 55, 302. [CrossRef]

42. Collell, G.; Fauquet, J. Brain activity and cognition: A connection from thermodynamics and information theory. Front. Psychol. 2015, 6, PMC4468356. [CrossRef] [PubMed]

43. Tsallis, A.C.; Tsallis, C.; Magalhaes, A.C.N.; Tamarit, F.A. Human and computer learning: An experimental study. Complexus 2003, 1, 181-189. [CrossRef]

44. Tsallis, C. Possible generalization of Boltzmann-Gibbs statistics. J. Stat. Phys. 1988, 52, 479-487. [CrossRef] 